\title{
Lettre à la rédaction : Le phénazépam utilisé comme arme chimique ? Discrimination par l'analyse des cheveux
}

\section{Hair as a discrimination tool in an homicide involving phenazepam}

Pascal KINTZ ${ }^{(1) *}$, Marion VILLAIN ${ }^{(1)}$, Christian STAUB ${ }^{(2)}$

(1) Laboratoire ChemTox, Illkirch - FRANCE

(2) Laboratoire Institut de Médecine Légale, Genève - SUISSE

*Auteur à qui adresser la correspondance ; Pascal KINTZ, Laboratoire ChemTox, 3, rue Gruninger, Parc d'Innovation - 67400 Illkirch-Graffenstaden - FRANCE Tél : +33 (0)3904033 32 - Fax : +33 (0)39040 0541 - E-mail : pascal.kintz@wanadoo.fr

(Reçu le 7 juillet 2004 ; accepté après modifications le 8 octobre 2004)

La place majeure des cheveux dans l'expertise judiciaire n'est plus à démontrer. En moins de 10 ans, cette matrice s'est imposée, en particulier lors de l'interprétation médico-légale (1). A ce titre, les cheveux ou les autres poils font désormais partie des prélèvements obligatoires dans le cadre de la bonne exécution des analyses (2).

La fenêtre de détection des xénobiotiques a pu être complètement modifiée par l'introduction du cheveu dans l'arsenal analytique. Cette matrice possède la propriété unique d'être le marqueur des expositions répétées ou chroniques, permettant en outre d'établir le profil de consommation à long terme et son évolution. Dans le cadre de la toxicologie postmortem, l'analyse sanguine ou urinaire et l'analyse des cheveux s'avèrent plutôt complémentaires, le sang ou les urines permettant de caractériser un usage ponctuel et les cheveux une exposition cumulée. Plus récemment, du fait d'ou- tils plus sensibles, une exposition unique à un agent chimique (benzodiazépines, hypnotiques, neuroleptiques) a pu être caractérisée dans les cheveux, en particulier dans le cadre de la soumission chimique.

L'analyse du sang d'autopsie permet de mettre en évidence la trace de tout xénobiotique circulant au moment du décès et donc d'établir le cas échéant une altération de la vigilance ou l'imputabilité du décès à un ou plusieurs toxiques qui auraient été retrouvés à une concentration potentiellement létale. Dans ces conditions, il arrive souvent qu'une substance psychoactive soit identifiée dans le sang et que se pose la question de savoir si cette substance était régulièrement consommée par la victime ou qu'au contraire, elle ait été administrée par l'agresseur afin de sédater sa future victime avant le passage à l'acte; dans ce dernier cas, la notion de préméditation paraît probable. 
Nous rapportons ici le cas d'un homme tué à coups de couteau et dont l'analyse du sang a révélé la présence d'une benzodiazépine. Le magistrat en charge de l'affaire ayant souhaité savoir si l'intéressé prenait régulièrement ce type de médicament, les cheveux ont été utilisés pour leur pouvoir discriminant.

\section{Circonstances du décès et prélèvement}

Un homme est retrouvé mort, tué à coups de couteau. Son épouse pourrait être impliquée dans cet homicide. Dans le sang, prélevé chez la victime, nous avons mis en évidence du phénazépam, une benzodiazépine anxiolytique. Une mèche de cheveux de la victime a été prélevée lors de l'autopsie et conservée à température ambiante. Notre laboratoire a reçu l'échantillon environ 1 mois après. Il est constitué d'une petite mèche de $4 \mathrm{~cm}$, correctement orientée.

Le phénazépam nous a été fourni par le Dr Peter Iten de l'IML de Zurich.

\section{Analyse des cheveux}

Après décontamination par le dichlorométhane ( 2 fois $5 \mathrm{ml}$, pendant $2 \mathrm{~min}$ ), la mèche a été coupée en $2 \mathrm{seg}$ ments (le faible échantillon ne permettait pas une segmentation plus courte) : de la racine à $1 \mathrm{~cm}$, et de 1 à $4 \mathrm{~cm}$, afin d'observer une éventuelle variation de concentration. Chaque segment a ensuite été coupé en fragments inférieurs à $1 \mathrm{~mm}$, puis 20 à $30 \mathrm{mg}$ ont été incubés à $40^{\circ} \mathrm{C}$ pendant $16 \mathrm{~h}$ dans $1 \mathrm{ml}$ de tampon phosphate à $\mathrm{pH} 8,4$, en présence de 1 ng de diazépam$\mathrm{d}_{5}$, utilisé comme étalon interne. Après une extraction par $5 \mathrm{ml}$ de dichlorométhane/éther diéthylique (80/20, $\mathrm{v} / \mathrm{v}$ ) et évaporation à $\mathrm{sec}$, le résidu a été reconstitué dans $50 \mu \mathrm{l}$ d'acétonitrile/eau $(50 / 50, \mathrm{v} / \mathrm{v})$. Un aliquot de $10 \mu \mathrm{l}$ a été injecté dans une colonne XTerra C18 (100 x $2,1 \mathrm{~mm}, 3,5 \mu \mathrm{m}$ ) d'un système Alliance 2695 (Waters St Quentin-en-Yvelines). La phase mobile $(0,2 \mathrm{ml} / \mathrm{min})$ était de type binaire acétonitrile/tampon formiate ( 2 $\mathrm{mM}, \mathrm{pH} 3,0)$. Les analytes ont été identifiés par spectrométrie de masse en tandem sur un système Electrospray Waters Quattro Micro en mode positif. Les conditions suivantes ont été utilisées : tension de capillaire $1,0 \mathrm{kV}$, température de la source $120^{\circ} \mathrm{C}$, température du gaz de désolvatation $350^{\circ} \mathrm{C}$ avec un débit de $550 \mathrm{l} / \mathrm{h}$, et pression d'argon $3 \times 10^{-3} \mathrm{Bar}$. Le phénazépam (tension de cône à $40 \mathrm{~V}$ ) a été identifié et dosé par les transitions m/z 351,0 à 186,1 (32 eV) et 351,0 à 206,2 $(38 \mathrm{eV})$. Le diazépam-d $\mathrm{d}_{5}$ (tension de cône à
$60 \mathrm{~V}$ ) était suivi et quantifié par les transitions $\mathrm{m} / \mathrm{z}$ 290,1 à $154,1(30 \mathrm{eV})$ et 290,1 à 198,2 (30 eV).

\section{Résultats et discussion}

Dans les conditions chromatographiques décrites, il n'y avait pas d'interférence entre les analytes et des substances endogènes présentes dans les cheveux.

La limite de détection a été évaluée à $2 \mathrm{pg} / \mathrm{mg}$ à partir d'un échantillon de $20 \mathrm{mg}$. La linéarité de la méthode a été vérifiée de 5 à $100 \mathrm{pg} / \mathrm{mg}\left(\dot{\mathrm{r}}^{2}=0,998\right)$. La précision ( 8 échantillons de cheveux blancs surchargés à une concentration finale en phénazépam de $20 \mathrm{pg} / \mathrm{mg}$ et analysés le même jour) a été mesuréeè à $14,6 \%$.

L'analyse des cheveux de la victime a donné les résultats suivants:

- segment 0 à $1 \mathrm{~cm}: 58 \mathrm{pg} / \mathrm{mg}$

- segment 1 à $4 \mathrm{~cm}: 32 \mathrm{pg} / \mathrm{mg}$

Le chromatogramme du premier segment est représenté Figure 1.

Ces résultats démontrent sans aucune ambiguiité que la victime avait été exposée au phénazépam à plusieurs reprises dans le passé, sans qu'on puisse y rattacher une quelconque notion quantitative. A titre de comparaison, la femme de la victime qui a affirmé prendre 1 à 2 comprimés par semaine avait les concentrations suivantes dans les cheveux : segment 0 à $1 \mathrm{~cm}, 123 \mathrm{pg} / \mathrm{mg}$; segment 1 à $2 \mathrm{~cm}, 63 \mathrm{pg} / \mathrm{mg}$ et segment 2 à $4 \mathrm{~cm}$, $31 \mathrm{pg} / \mathrm{mg}$.

Le phénazépam (fénazépam, 7-bromo-5-(2-chlorophenyl)-1,3-dihydro-2H-1,4-benzodiazepin-2-one) est une benzodiazépine anxiolytique commercialisée en Ukraine et en Russie sous forme de comprimés blancs dosés à 0,5 ou $1 \mathrm{mg}$. Très peu de données sont disponible sur le produit, tout au plus sait-on qu'il possède des propriétés sédatives supérieures à celles du chlordiazépoxide ou du diazépam et qu'il a une activité anticonvulsivante équivalente à celle du clonazépam $(3,4)$.

\section{Conclusion}

L'analyse des cheveux, pratiquée par CLHP-SM/SM a permis de mettre en évidence des concentrations faibles de phénazépam, mais indicatives d'une exposition répétée. La préméditation semble difficile à retenir. Le résultat complète donc ceux obtenus dans le sang. Ce type d'investigation, réalisée dans le cadre d'une expertise judiciaire sur des échantillons postmortem, renforce la place majeure des cheveux dans l'interprétation des observations médico-légales et confirme à nouveau tout l'intérêt, même à titre conservatoire, de prélever au moment de l'autopsie des phanères. 


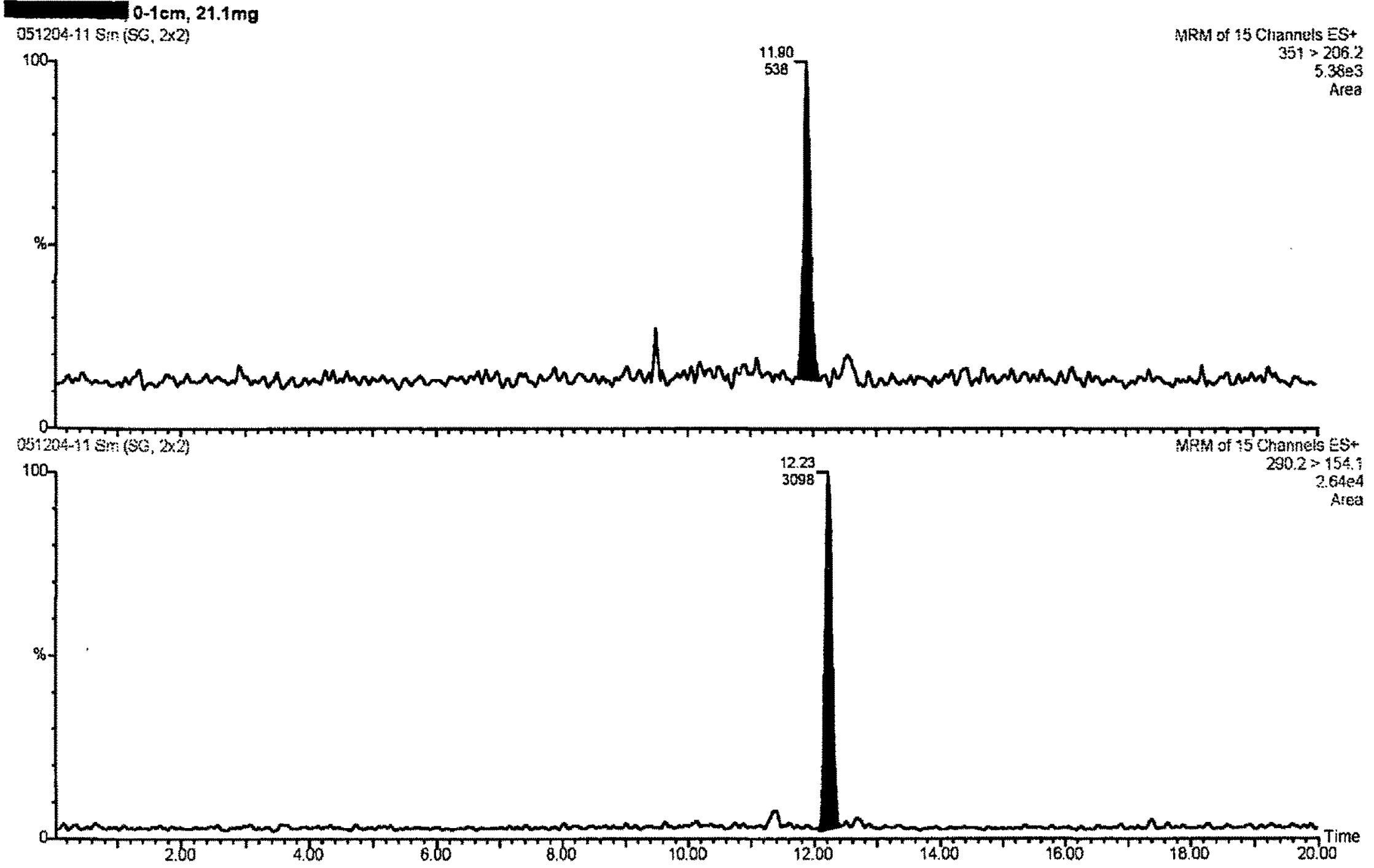

Figure 1 : Chromatogramme du segment de 0 (racine) à $1 \mathrm{~cm}$, avec les transitions de quantification $\mathrm{m} / \mathrm{z} 351,0$ à 206,2 (phénazépam à $58 \mathrm{pg} / \mathrm{mg}$ ) et 290,2 à 154, I (diazépam $\left.-d_{5}\right)$.

\section{Références}

1. Kintz P. Value of hair analysis in postmortem toxicology. Forensic Sci. Int. $2004 ; 142: 127-34$.

2. Kintz P. Toxicologie judiciaire. Quels prélèvements ? Quelles techniques analytiques? J. Med. Leg. Droit Med. $2003 ; 46: 233-7$.

3. Zherdev V.P., Martynova L.A., Caccia S., Garattini S., Ekonomov A.L. Pharmacokinetics of phenazepam. Farmakol. Toksikol. $1985 ; 48: 31-5$.

4. Voronina T.A., Garibova T.L., Nerobkova L.N. Characteristics of the anticonvulsant effects of phenazepam. Farmakol. Toksikol. $1980 ; 43: 546-51$. 